\title{
Microrheometric upconversion-based techniques for intracellular viscosity measurements
}

\author{
Paloma Rodríguez-Sevilla, ${ }^{1}$ Yuhai Zhang, ${ }^{2}$ Nuno de Sousa, ${ }^{3,4}$ Manuel I. Marqués, ${ }^{5}$ Francisco Sanz- \\ Rodríguez, ${ }^{1,6}$ Daniel Jaque, ${ }^{1,6}$ Xiaogang Liu, ${ }^{2}$ and Patricia Haro-González ${ }^{1}$
}

${ }^{1}$ Fluorescence Imaging Group, Departamento de Física de Materiales, Universidad Autónoma de Madrid, 28049 Madrid, Spain

2 Department of Chemistry, National University of Singapore, Science Drive 3, Singapore 117543 , Singapore

${ }^{3}$ Departamento de Física de la Materia Condensada, Condensed Matter Physics Center (IFIMAC), and Nicolás Cabrera Institute, Universidad Autónoma de Madrid, 28049 Madrid, Spain

${ }^{4}$ Donostia International Physics Center (DIPC), Donostia-San Sebastián 20018, Spain

${ }^{5}$ Departamento de Física de Materiales, Condensed Matter Physics Center (IFIMAC), and Nicolás Cabrera Institute, Universidad Autónoma de Madrid, 28049 Madrid, Spain

${ }^{6}$ Instituto Ramón y Cajal de Investigaciones Sanitarias, Hospital Ramón y Cajal, Madrid 28034, Spain

\begin{abstract}
Rheological parameters (viscosity, creep compliance and elasticity) play an important role in cell function and viability. For this reason different strategies have been developed for their study. In this work, two new microrheometric techniques are presented. Both methods take advantage of the analysis of the polarized emission of an upconverting particle to determine its orientation inside the optical trap.

Upconverting particles are optical materials that are able to convert infrared radiation into visible light. Their usefulness has been further boosted by the recent demonstration of their three-dimensional control and tracking by single beam infrared optical traps. In this work it is demonstrated that optical torques are responsible of the stable orientation of the
\end{abstract}

Optical Trapping and Optical Micromanipulation XIV, edited by Kishan Dholakia, Gabriel C. Spalding, Proc. of SPIE Vol. 10347, 103471S · @ 2017 SPIE · CCC code: 0277-786X/17/\$18 · doi: 10.1117/12.2275944 
upconverting particle inside the trap. Moreover, numerical calculations and experimental data allowed to use the rotation dynamics of the optically trapped upconverting particle for environmental sensing. In particular, the cytoplasm viscosity could be measured by using the rotation time and thermal fluctuations of an intracellular optically trapped upconverting particle, by means of the two previously mentioned microrheometric techniques.

Keywords: Microrheometry, optical trapping, upconverting particle, optical torque, intracellular viscosity

\section{INTRODUCTION}

Cellular rheological parameters (viscosity, elasticity and creep compliance) role the different processes that take place inside the cell. For example, viscosity controls the diffusion of molecules and organelles. ${ }^{1,2}$ Moreover, such parameters are an indicative of the cell state, allowing to determine the appearance and onset of diseases. ${ }^{3}$ Because of that, rheological parameters are of great interest, and a wide variety of studies have focused on their characterization. ${ }^{1,}$ For such purpose, lots of different rheometric techniques have been developed. They can be classified in passive and active methods. Passive techniques are based on the measurement of the thermal fluctuations of a probe, which could be endogenous (organelle, vesicle or molecule) or exogenous (internalized particle o molecule). ${ }^{1,5-7}$ The Brownian motion of the probe could be tracked by using video-based methods or by the analysis of its emission, in the case of luminescent probes. ${ }^{5,8-10}$ On the other hand, active techniques exert a force over the cell to test its response to the applied stimulus. ${ }^{11,}$ 12 The action could be performed directly over the cell or by using a probe (usually polystyrene or silica beads). For example, magnetic rheometers have been used for the determination of the viscoelastic character of the cytoplasm of living cells. ${ }^{13}$ The spinning of cylindrical particles, induced by the action of an external magnetic field, have been used to measure the intracellular viscosity. This study evidences the usefulness of micromotors to test intracellular rheological parameters. Active techniques should exert forces on the range of piconewtons in order to not compromise cell viability. In this sense, optical trapping (OT) has been evidenced as the perfect tool for such purpose, since the strength of optical forces lie in that range. ${ }^{14,15}$ OT takes advantage of the optical force and torque produced by the light-matter interaction that stably trap the particle in a certain orientation inside the optical trap (i.e. focused laser beam). ${ }^{16}$ Optical forces are responsible for the confinement of the particle within the beam focus, while optical torques drive a rotation of the particle towards the stable orientation. Thus, based on this premise, optically driven motors can be also used as rheometers. ${ }^{17}$ For such purpose, the real time orientation of the rotating particle must be characterized. If the particle is large enough, video-based techniques can be used. However, in most of the cases, the size of the probe is reduced in order to enhance spatial resolution, thus they cannot be optically resolved. Therefore an alternative approach is mandatory. As introduced above, luminescent particles can be used as sensor agents. In particular, upconverting particles (UCPs) have been widely used in different biological studies. ${ }^{18-21}$ They present the capability to produce high energy radiation (ultraviolet or visible) after the absorption of low energy photons (infrared), through a process known as upconversion. ${ }^{22}$ This ability have been already proven to be of great interest for the development of cancer treatments, 
such as the photodynamic therapy or controlled drug release. ${ }^{23-26}$ Moreover, the upconversion luminescence of nonspherical UCPs is polarized. ${ }^{27,28}$ This leads to a dependence of the upconversion luminescence on the orientation of the particle. ${ }^{29}$ Thus, the analysis of the UCP luminescence can be used to monitor its orientation during the optically-driven rotation.

Based on the previous idea, two microrheometric techniques are here presented. Both methods take advantage of the analysis of the polarized emission to determine the orientation of the upconverting particle inside the optical trap. The active technique relies on the laser-induced rotation of trapped hexagonal upconverting particles to test the dynamic viscosity of the cytoplasm. On the other hand, the passive method takes advantage of the thermal vibration of the trapped particle when it reaches its stable orientation inside the optical trap.

\section{SAMPLE AND EXPERIMENTAL SETUP}

$\beta-\mathrm{NaYF}_{4}: \mathrm{Er}^{3+}, \mathrm{Yb}^{3+} \mathrm{UCPs}$ were synthetized by a hydrothermal procedure as explained elsewhere. ${ }^{30}$ These particles present a hexagonal shape, as shown in Figure 1a, with a mean diameter of $800 \mathrm{~nm}$ and a thickness of $400 \mathrm{~nm}$. As mentioned in the introduction, they present polarized emission which have been characterized by using the experimental setup depicted in Figure 1b. It is composed by a modified confocal microscope which allows to both spectroscopic characterization and optical manipulation of the particles. Briefly, the radiation coming from a fiber-coupled diode laser is focused by using a microscope objective. This radiation is used for excitation of the particle luminescence and its optical manipulation. The luminescence of the particle is collected with the same microscope objective and directed towards a high sensitive Si charge-coupled device camera (Synapse, Horiba), attached to a monochromator (iH320, Horiba), for its real time analysis. For polarized spectroscopy, a linear polarizer (LPNIR050-MP2, Thorlabs) was placed at the entrance of the detector to select the polarization state of the luminescence. As a results of the upconversion process, $\beta-\mathrm{NaYF}_{4}: \mathrm{Er}^{3+}, \mathrm{Yb}^{3+} \mathrm{UCPs}$ showed green and red upconversion emission when excited with $980 \mathrm{~nm}$ radiation. ${ }^{31}$ Only red emission were analyzed, since this emission band was evidenced to have a higher polarization degree than the green emission band. ${ }^{29}$ Optical images of the optical trap area where recorded using a CMOS camera.

(a)

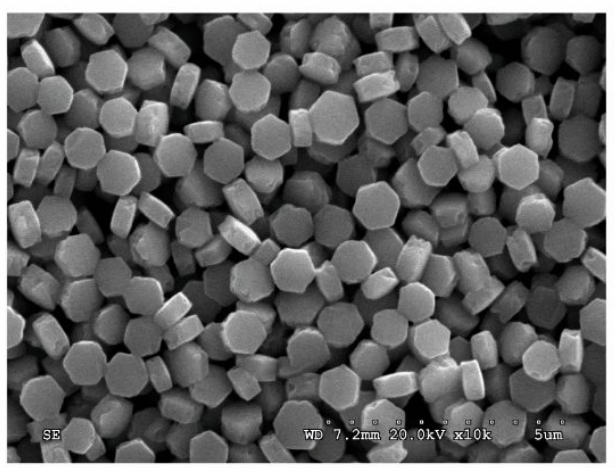

(b)

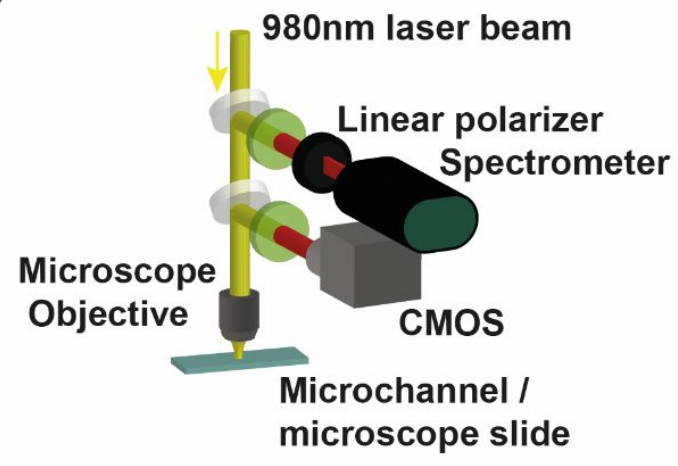

Figure 1. (a) SEM image of the $\beta-\mathrm{NaYF} 4: \mathrm{Er}^{3+}, \mathrm{Yb}^{3+}$ UCPs. (b) Schematic representation of the experimental setup. White and green discs represents mirrors and filters, respectively. Reprinted with permission from Nano Letters 2016, 16 (12), 8005-8014. Copyright 2016 American Chemical Society. 


\section{POLARIZED SPECTROSCOPY}

\section{1 Dried sample characterization}

A diluted suspension of the UCPs $\left(2.3 \times 10^{7} \mathrm{UCPs} / \mathrm{cm}^{3}\right)$ was dried on a microscope slide by drop casting and the luminescence of isolated particles were analyzed. The excitation/detection direction was perpendicular to the substrate in such a way that two different UCP configuration were assessed (see Figure 2a). In the horizontal configuration, the

(a)
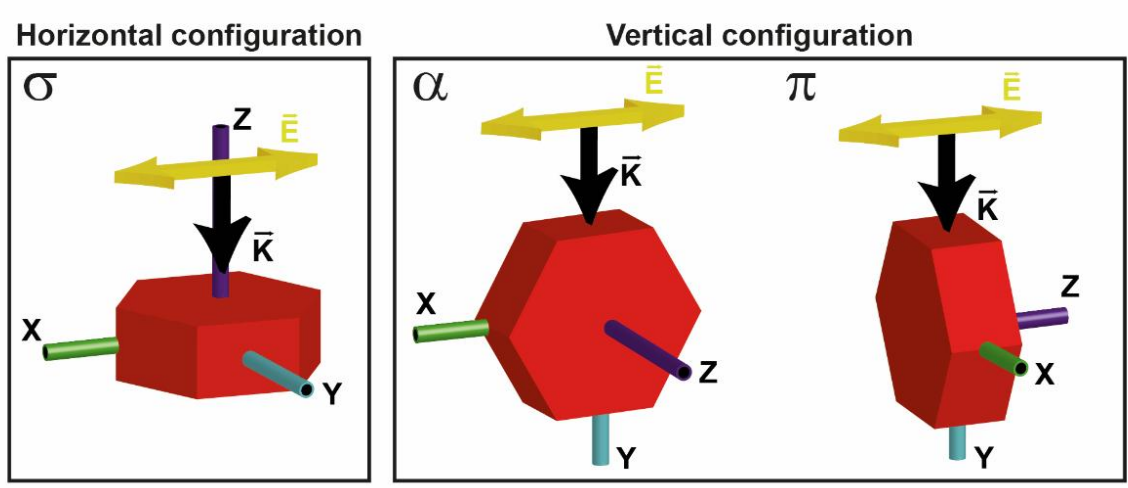

(b)
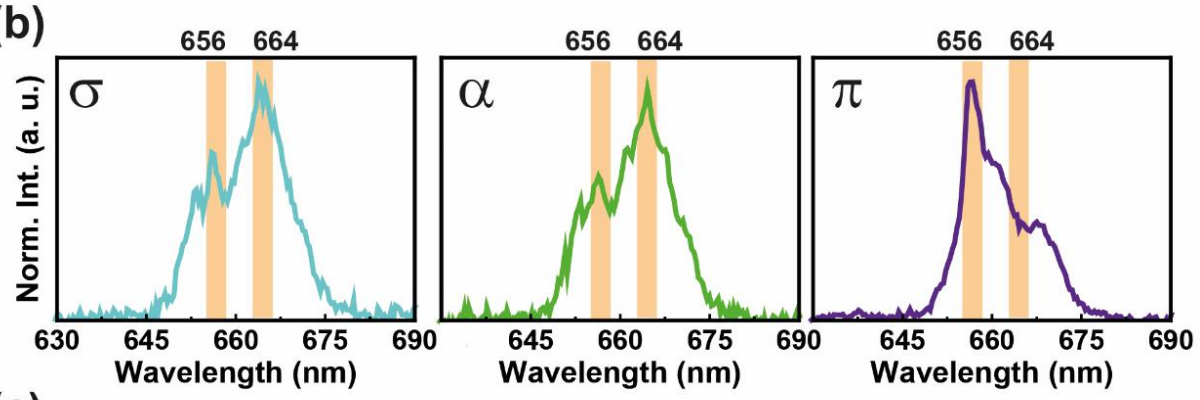

(c)
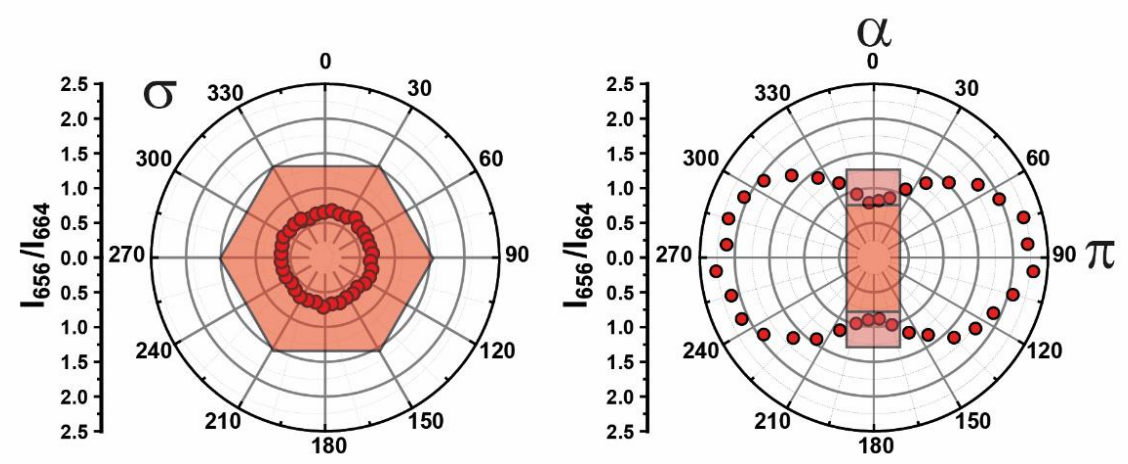

Figure 2. (a) Schematic representation of the two UCP configurations. The three main polarization states are included. The propagation direction of the light is indicated by vector $k$, while its polarization is represented by vector $E$. (b) Emission spectra for the three main polarization states. (c) Polar plots of the intensity ratio of the two peaks indicated in (b) as a function of the polarization state. Left for horizontal configuration, and right for vertical orientation. Reprinted with permission from Nano Letters 2016, 16 (12), 8005-8014. Copyright 2016 American Chemical Society. 
particle lies with its hexagonal facet perpendicular to the excitation/detection direction, while, in the vertical configuration, the longitudinal axis of the particle is parallel to the excitation/detection direction. Polarized spectra were analyzed for both particle orientation by rotating the linear polarizer and detecting the emission spectra for different polarization states. At this point we state that, based on a previously published work, ${ }^{27}$ the studied UCPs are uniaxial crystals with their optical axis perpendicular to their hexagonal facet. This assumption implies that the two orientations of the particle in respect to detection direction allow to assess three polarization states: $\sigma, \alpha$, and $\pi$. In the $\sigma$ polarization state (Figure 2a, left) the luminescence propagates along the optical axis (z axis) of the crystal and, consequently, with a polarization perpendicular to it. This polarization state corresponds to the particle being in the horizontal configuration. On the other hand, in the vertical orientation, light propagates perpendicularly to the optical axis of the crystal with a polarization that can be perpendicular ( $\alpha$, Figure 2a, center) or parallel ( $\pi$, Figure 2a, right) to it, thus $\alpha$ and $\pi$ polarization states are accessible. The emission spectra detected for the three polarization states are show in Figure $2 \mathbf{b}$. As can be seen, $\sigma$ and $\alpha$ polarization states are analogous, whereas $\pi$ polarization state present a completely different spectral shape. It is worth mentioning that this spectral modulation was expected for uniaxial crystals. In particular, the most significant spectral change take place at 656 and $664 \mathrm{~nm}$ emission peaks. For a better characterization of the spectral modulation, polar plots of the intensity ratio $\left(\mathrm{I}_{656} / \mathrm{I}_{664}\right)$ of these two peaks as a function of the polarization state are shown in Figure 2c. In the horizontal configuration (Figure 2c, left), the emission spectrum do not change with the polarization state since only $\sigma$ polarization state is accessible, thus a circular diagram is obtained. However, in the vertical orientation (Figure 2c, right), the emission spectrum changes from that associated to $\alpha$ polarization state to $\pi$ polarization state when the polarization state is detected perpendicularly or parallel to the optical axis of the UCP. Polar graphs in Figure 2c evidence that the orientation of the particle can be elucidated through the analysis of the luminescence spectra.

\subsection{Optical manipulation}

\subsubsection{Experimental results}

The former analysis can be used for the determination of the UCP orientation within the optical trap. It is expected that, when it is in the horizontal configuration, the non-polarized (no polarization state selected) emission spectrum is that associated to the $\sigma$ polarization state. However, when the particle is in the vertical orientation, the emission spectrum is a combination of that obtained for $\alpha$ and $\pi$ polarization states.

Based on this idea, the orientation of the UCPs within the optical trap was elucidated by analyzing the non-polarized emission spectrum of the trapped particle. For such purpose, a diluted aqueous suspension of particles $\left(2.3 \times 10^{7}\right.$ UCPs $/ \mathrm{cm}^{3}$ ) were injected into a microchannel ( $\mu$-Slide I 80106, Ibidi Inc.). Then, a single particle was optically trapped and its emission spectrum detected. Figure 3a shows the non-polarized spectrum obtained for a single optically trapped UCP. As can be seen, the spectral shape of the emission is a combination of that associated to $\alpha$ and $\pi$ polarization. This unequivocally indicates that the stable orientation within the optical trap is the vertical configuration, as schematically represented in the inset of Figure 3a. For a better characterization, the emission spectra as a function of the polarization

state was also analyzed. The polar plot of the intensity ratio is represented in Figure $\mathbf{3 b}$. As expected, the dependence of 
(a)

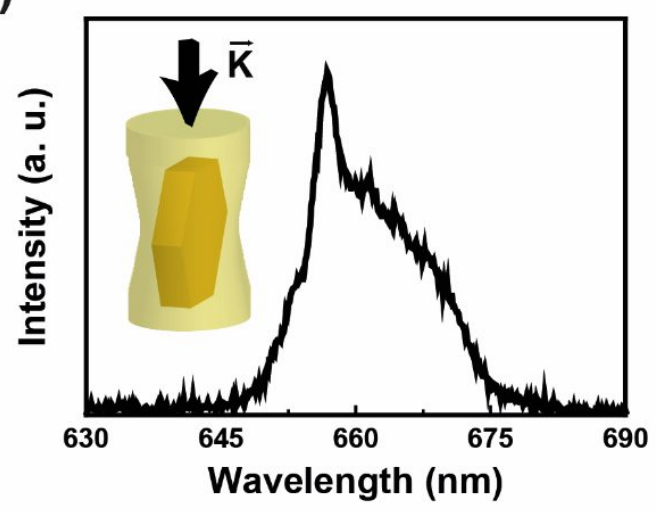

(b)

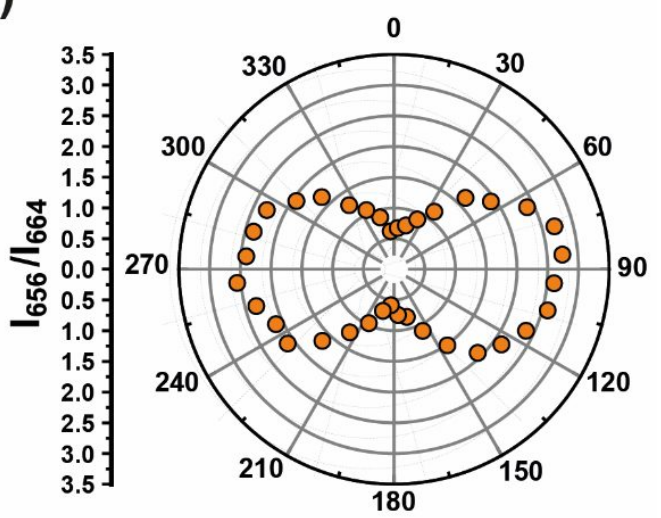

Figure 3. (a) Non-polarized spectrum (no polarization state is selected) obtained for an optically trapped UCP. Inset: Schematic representation of the stable orientation of the UCP inside the optical trap. (b) Polar diagram of the intensity ratio of $656 \mathrm{~nm}$ and $664 \mathrm{~nm}$ peaks as a function of the polarization state. Reprinted with permission from Nano Letters 2016, 16 (12), 8005-8014. Copyright 2016 American Chemical Society.

$\mathrm{I}_{656} / \mathrm{I}_{664}$ with polarization state is the same as that obtained for a UCP deposited on the vertical configuration (see Figure 2c, right). This further corroborates that UCPs stable orientate in the vertical configuration.

\subsubsection{Theoretical calculations}

The former results show that an optical torque is acting on the UCP. It induces a rotation of the particle around an axis perpendicular to the $\vec{k}$ vector of the trapping beam until the stable position (vertical configuration) is achieved for $\theta=$ $90^{\circ}$, being $\theta$ the angle between $\vec{k}$ vector and the optical axis of the UCNP. This rotation is schematically represented in Figure 4a. There are two possible rotations which lead to the same final orientation. The first one, (upper path) is a rotation around an axis parallel to the electric field of the trapping beam (axis $\mathrm{x}$ ) which it is characterized by the angle $\theta_{\mathrm{E}}$. In the second rotation (lower path), the rotation axis is parallel to the magnetic field (axis y) and it is represented by the angle $\theta_{\mathrm{H}}$. The optical torque acting on each rotation have been numerically calculated. To do that, numerical simulations of the interaction between the electromagnetic field and the single trapped UCP have been carried out based on the finite element method. ${ }^{32}$ In particular, a non-birefringent hexagonal particle, with a diameter of $800 \mathrm{~nm}$ and a thickness of $400 \mathrm{~nm}$, illuminated by a linearly polarized $980 \mathrm{~nm}$ plane wave has been considered. The optical torque, $\langle\vec{\Gamma}\rangle$, acting on the UCP was calculated by integrating the Maxwell stress tensor $\boldsymbol{T}$ over an arbitrary surface $\mathrm{S}$ enclosing the particle. ${ }^{33}$ The components of the Maxwell Stress tensor are given by:

$$
T_{i j}=\left[\epsilon_{0} \epsilon E_{i} E_{j}+\mu_{0} H_{i} H_{j}-\frac{1}{2}\left(\epsilon \epsilon_{0} E^{2}+\mu_{0} H^{2}\right) \delta_{i j}\right]
$$

where $\epsilon_{0}$ and $\epsilon$ are the vacuum and relative permittivity of the medium respectively, $\mu_{0}$ the magnetic permeability, $E_{i}$ and $H_{i}$ the $i$ component of electric and magnetic fields in the integration surface, $E$ and $H$ the absolute value of the electric and magnetic fields, and $\delta_{i j}$ is the Kronecker's delta. Once the Maxwell Stress tensor is calculated, the optical torque acting on the particle is obtained by computing: 
(a)
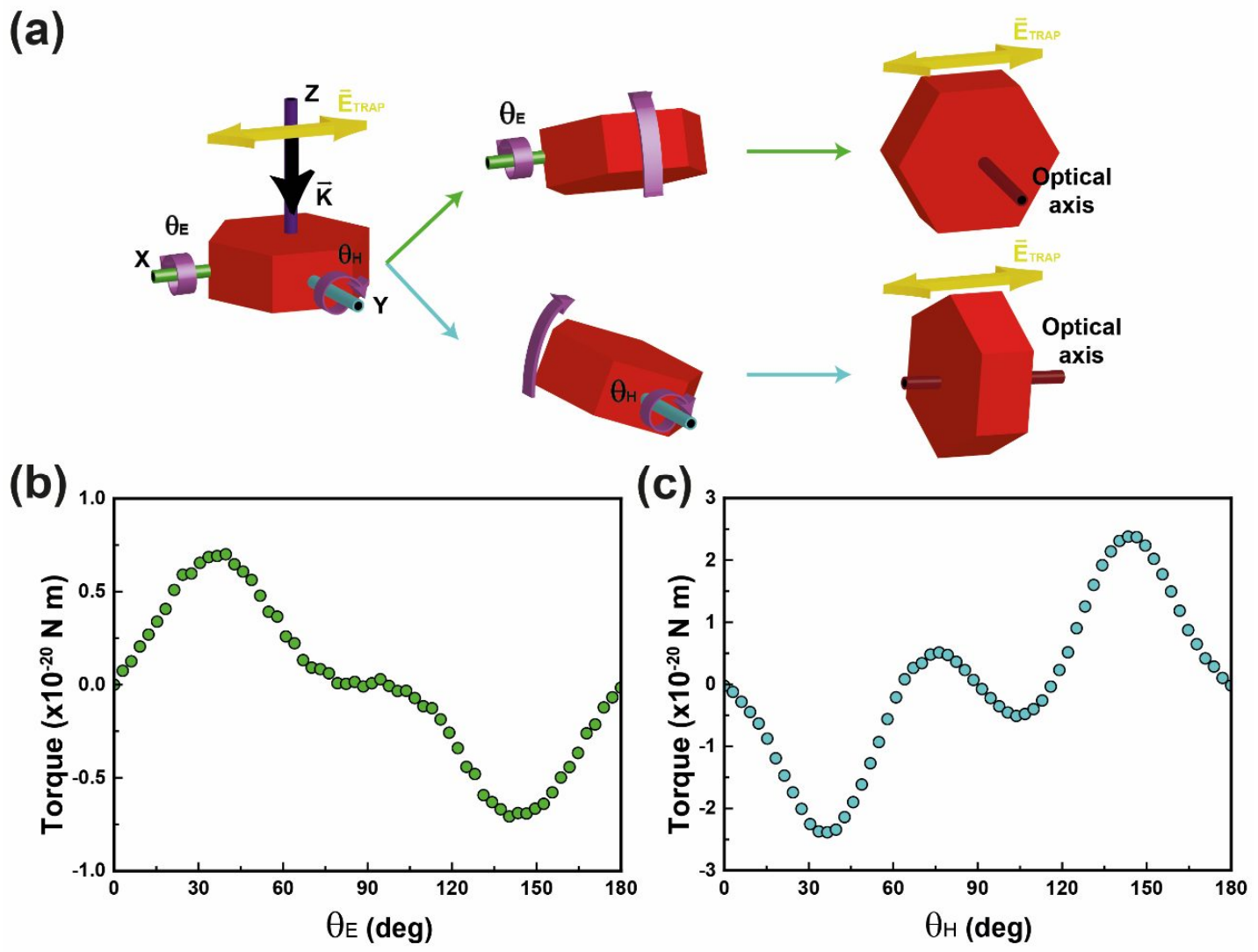

Figure 4. (a) Schematic representation of the transition from horizontal configuration to vertical orientation. Green upper path represents a rotation around an axis parallel to the electric field of the trapping light, while blue lower path shows a rotation around an axis parallel to the magnetic field. (b) Numerically calculated torque for the rotation around the electric field. (c) Numerically calculated torque for the rotation around the magnetic field. Reprinted with permission from Nano Letters 2016,16 (12), 8005-8014. Copyright 2016 American Chemical Society.

$$
\langle\vec{\Gamma}\rangle=-\left\langle\oint_{S} \hat{n}(\boldsymbol{T} \times \vec{r}) d \vec{S}\right\rangle
$$

with $\hat{n}$ the normal vector to the integration surface. Results for the two rotations depicted in Figure 4a are shown in Figure 4b-c. The stable orientation is reached when the optical torque has a null value and its first derivative is negative. This condition is accomplish by both torques for an angle $\theta_{\mathrm{E}}=\theta_{\mathrm{H}}=90^{\circ}$. However, for the rotation around an axis parallel to the magnetic field, $\theta_{\mathrm{H}}=0^{\circ}$ is also a stable orientation. This result demonstrates that the rotation around an axis parallel to the electric field is more likely to happen since for $\theta_{\mathrm{E}}=0^{\circ}$ the particle is not stably orientated. Thus, being the particle in the horizontal configuration the particle will flip to the vertical orientation by the action of a torque which rotation axis is parallel to the electric field of the trapping plane wave (Figure 4a, upper part, and Figure $4 \mathbf{b}$ ).

\section{MICRORHEOMETRIC TECHNIQUES}

The laser-induced rotation of the UCPs have been experimentally and numerically demonstrated in the previous section. The rotation velocity will depend on the characteristics of the medium where the UCP is immersed, at the same time as 
on the applied laser power (i.e. the magnitude of the optical torque). When there is a relative velocity between the particle and the fluid, a friction force appears which opposes to the movement of the particle. This drag force is proportional to the drag coefficient of the particle which depends on the viscosity of the fluid. This relation between the rotation velocity of the particle and the dynamic viscosity of the medium can be used for the determination of this latter if the laser-induced rotation of the UCP is tracked. Once in the vertical orientation, the UCP will start to oscillate around the stable orientation due to Brownian motion. Information about the static viscosity of the medium can be also obtained from these thermal fluctuations.

These two different situations are the base of the two developed microrheometric techniques: the active and the passive methods.

\subsection{Active method}

This method is based on the laser-induced rotation of the UCP. In a first order approximation, the equation that describes the rotation of the particle can be written as:

$$
\beta \frac{d \theta}{d t}=\langle\Gamma\rangle
$$

Where $\beta=6 \eta V f / f_{0}$ is the rotational friction coefficient of the UCP, with $f / f_{0}$ the Perrin friction factor, $\eta$ the dynamic viscosity of the medium, and $V$ the volume of the particle. ${ }^{34}\langle\Gamma\rangle$ is the torque calculated by using expression 2 . Thus, the transition time ( $\Delta t_{R}$, the total time the particle needs to go from the horizontal configuration to the vertical orientation) is:

$$
\Delta t_{R}=\beta \int_{0}^{\pi / 2} \frac{1}{\langle\Gamma\rangle} d \theta
$$

The magnitude of the optical torque as a function of the applied laser power density can be calculated and introduced in expression 4. Then, the dynamic viscosity of the medium as a function of the transition time and the applied laser power density $\left(I_{L}\right)$ is:

$$
\eta \approx 6.7 \times 10^{-14} I_{L} \Delta t_{R}
$$

Expression 5 shows that, in the active method, the dynamic viscosity of the medium in obtained through the measurement of the transition time of the particle. This transition time is obtained from the analysis of the emission spectra of the rotating UCP. Figure 5a shows a representative graph of the evolution of the intensity ratio $\mathrm{I}_{656} / \mathrm{I}_{664}$ as a function of time for a particle that is forced to rotate by the laser action. The three different states (horizontal configuration, transition, and vertical orientation) are clearly characterized by the value of $\mathrm{I}_{656} / \mathrm{I}_{664}$. Thus, for each rotation particle, the evolution of the emission spectrum is measured and $\mathrm{I}_{656} / \mathrm{I}_{664}$ calculated. Then, $\Delta t_{R}$ is obtained and the dynamic viscosity can be determines through expression 5 .

\subsection{Passive method}

As can be seen in Figure 5a, when the particle reaches the vertical orientation, it starts to oscillate around the stable 
(a)

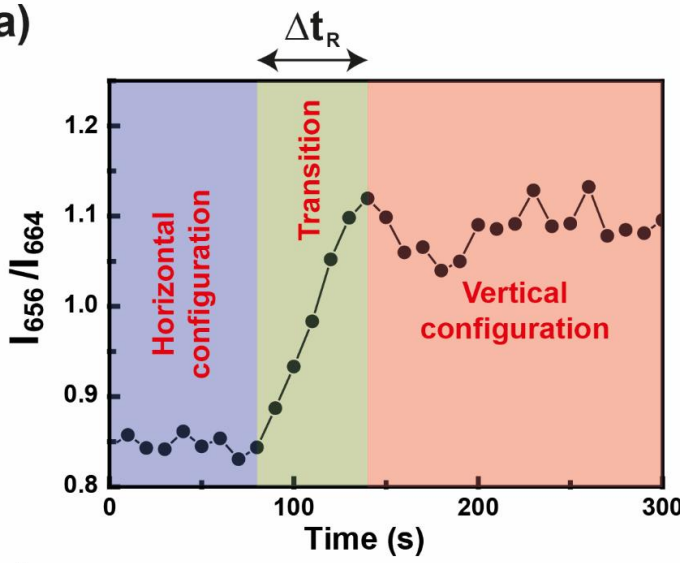

(c)

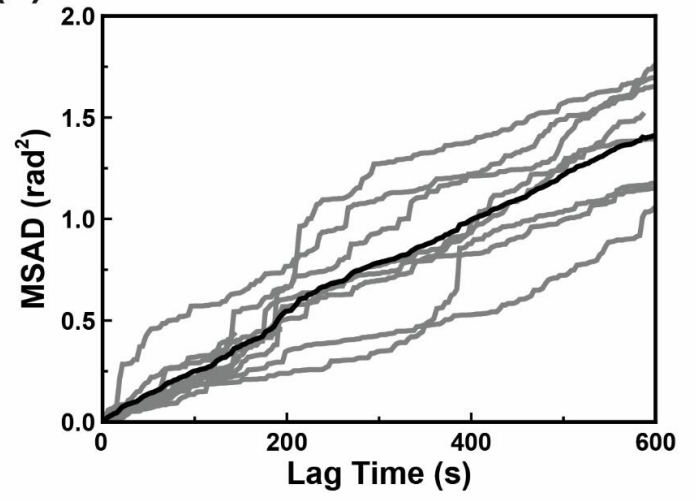

(b)

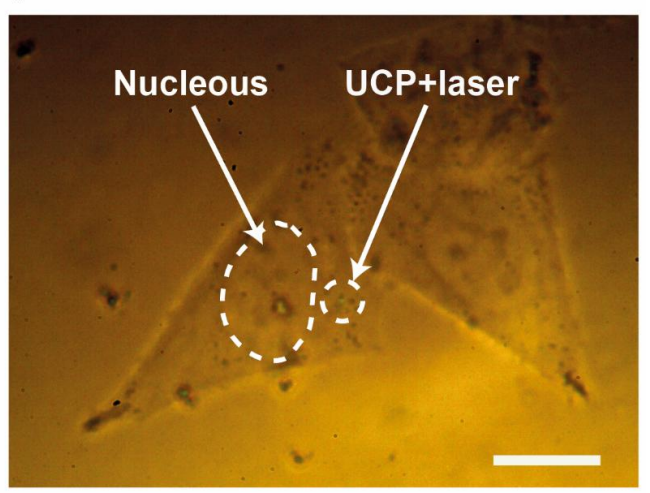

(d)

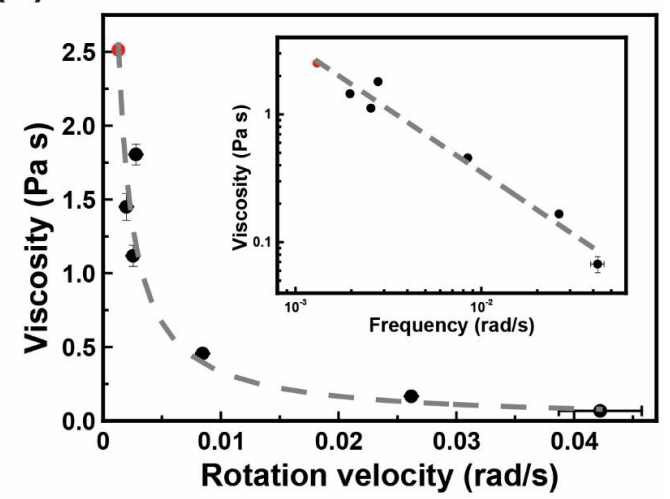

Figure 5. (a) Representative of the evolution with time of the intensity ratio measured for a rotating particle. (b) Representative optical image of a HeLa cell where an interiorized particle is indicated. Scale bare correspond to $30 \mu \mathrm{m}$. (c) MSAD as a function of the lag time. Gray lines correspond to individual measurements, whereas black line represents the mean of all of them. (d) Measures viscosity values as a function of the rotation velocity. Red dot correspond with the main value obtained from the passive method. Insets shows the log-log plot. Reprinted with permission from Nano Letters 2016, 16 (12), 8005-8014. Copyright 2016 American Chemical Society.

orientation, as the fluctuation of the intensity ratio evidence it. As commented above, these thermal oscillations can be used for the determination of the static viscosity of the fluid. The passive method is based on the analysis of the mean square angular displacement (MSAD) as a function of the lag time $(\tau):^{35}$

$$
M S A D=2 D \tau
$$

Where $D=k_{B} T / \beta$ is the diffusion coefficient of the particle, with $k_{B}$ the Boltzmann constant and $T$ the temperature. For the calculation of MSAD, the evolution of $\theta$ with time is needed. It is obtained from the values of $\mathrm{I}_{656} / \mathrm{I}_{664}$. When the particle is in its front configuration (rotation angle $\left.\theta=0^{\circ}\right), \mathrm{I}_{656} / \mathrm{I}_{664}$ reaches its minimum value $\left(\mathrm{I}_{656} / \mathrm{I}_{664}(0)=\mathrm{I}_{656} / \mathrm{I}_{664}{ }^{\mathrm{min}}\right)$, which is the pure intensity ratio value characteristic of the $\sigma$ polarization state. On the other hand, when the particle is in the vertical configuration, the intensity ratio calculated from the non-polarized emission spectrum would result from the average of the $\mathrm{I}_{656} / \mathrm{I}_{664}$ values characteristic of both $\alpha$ and $\pi$ polarizations. In this case, $\mathrm{I}_{656} / \mathrm{I}_{664}$ acquires its maximum 
value $\left(\mathrm{I}_{656} / \mathrm{I}_{664}(\pi / 2)=\mathrm{I}_{656} / \mathrm{I}_{664}{ }^{\max }\right)$. For intermediate configurations, the intensity ratio calculated from non-polarized emission spectra would have values between $\mathrm{I}_{656} / \mathrm{I}_{664}{ }^{m i n}$ and $\mathrm{I}_{656} / \mathrm{I}_{664}{ }^{m a x}$. In particular, for any giving angle, the nonpolarized emission would account for $\pi$ and $\alpha$ contributions that would be weighted by the sine and cosine of the rotation angle. Therefore, for any rotation angle $\theta$, the intensity ratio can be written as:

$$
I_{656} / I_{664}(\theta)=I_{656} / I_{664}{ }^{\min } \operatorname{sen}^{2} \theta+I_{656} / I_{664}{ }^{\max } \cos ^{2} \theta
$$

Then, the rotation angle is given by:

$$
\theta\left(I_{656} / I_{664}\right)=\operatorname{acos}\left(\sqrt{\frac{I_{656} / I_{664}{ }^{\max }-I_{656} / I_{664}}{I_{656} / I_{664}{ }^{\max }-I_{656} / I_{664}}}\right)
$$

Therefore, the angular displacement (i.e. the evolution of $\theta$ ) can be obtained from the measured $\mathrm{I}_{656} / \mathrm{I}_{664}$. Then, the value of MSAD as a function of time can be computed. Finally, by fitting the value of MSAD vs lag time to expression 6 , the static viscosity can be obtained from the slope of the curve.

\section{INTRACELLULAR MICRORHEOMETRY}

The intracellular viscosity of HeLa cells was measured by using the two methods described in the previous section. For such purpose, HeLa cancer cells were incubated with a suspension of UCPs in such a way that, as a results of an endocytic process, some of the UCPs were incorporated into the cells (see optical image in Figure 5b). Up to 34 different measurements were performed over more than 20 different HeLa cells.

Firstly, the static intracellular viscosity was measured. In this case, only particles in the vertical orientation were used. Figure 5c shows a set of MASDs as a function of the lag time obtained for a total number of 10 particles. Gray lines correspond to the individual measurements, whereas the red line corresponds to the averaged of all of them. For each measurement, a value for the viscosity could be obtained by fitting the MSAD curve to expression 6 . The average of all measurements gives a mean viscosity of $2.51 \pm 0.01 \mathrm{~Pa}$ s. This value is in good agreement with those previously reported HeLa cells. ${ }^{36,37}$

For the determination of the dynamic viscosity by using the active methods, UCPs in horizontal configuration were used. Figure 5a shows, as a representative example, the time evolution of the intensity ratio $\mathrm{I}_{656} / \mathrm{I}_{664}$ as obtained for an intracellular UCP optically trapped with a power density of $4.1 \times 10^{10} \mathrm{~W} / \mathrm{m}^{2}$. Under these conditions, experimental data reveals a transition time of $\Delta \mathrm{t}_{\mathrm{R}}=60 \pm 10 \mathrm{~s}$ ( or $\omega_{\mathrm{R}}=2.78 \times 10^{-3} \pm 0.02 \times 10^{-3} \mathrm{rad} / \mathrm{s}$ ). This, according to expression 5 , leads to an intracellular dynamic viscosity, for this particular frequency, of $\eta\left(2.78 \times 10^{-3} \mathrm{rad} / \mathrm{s}\right)=170 \pm 30 \mathrm{mPa} \cdot \mathrm{s}$. The same experimental and analytical procedure was performed over a total number of 6 different UCPs located in different HeLa cells and by using different laser trapping intensities. The obtained viscosity values and the corresponding rotation frequencies are shown in Figure 5d (black dots). In this graph, the red dot corresponds to the mean viscosity value obtained from the passive measurements. The rotation frequency for that value is estimated by dividing the accumulated rotated angle by the elapsed time: $1.18 \mathrm{rad}$ in $600 \mathrm{~s}$, leads to an effective rotation frequency close to $0.002 \mathrm{rad} / \mathrm{s}$. Inset in Figure 5d shows the linear dependence with a slope around -1 in a $\log -\log$ representation. The obtained results reveal that the intracellular viscosity of HeLa cells has a mean static value of around $2.5 \mathrm{~Pa}$ s which decreases monotonously 
with increasing frequency. From the $\log -\log$ representation we can conclude that $\eta \sim 1 / \omega_{R}$. This fact points out that, in this range of frequencies, the cytoplasm behaves as a viscous liquid, as it was demonstrated by former studies. ${ }^{1,13}$

\section{CONCLUSIONS}

Two different rheometric techniques have been used for the determination of the intracellular viscosity of HeLa cells. Both methods are based on the determination of the orientation of a single optically trapped upconverting particle. The polarized emission presented by this type of crystals has allowed to determine the laser-induced rotation frequency of the particles and the fluctuations of their orientation produced by Brownian motion. This strategy permits the use of nonoptically resolved particles which sizes can enhance the spatial resolution of the measurements or increase the internalization rate of the particles inside the cells.

Finally, this work evidences the potential of the combination of optical trapping with single particle spectroscopy. New techniques can be developed based on those here presented which can be of great interest many different fields such as biomedicine and food industry.

\section{ACKNOWLEDGEMENTS}

This work was supported by the Spanish Ministerio de Educación y Ciencia (MAT2016-75362-C3-1-R) and by COST Action 1403. P.H.G. thanks the Spanish Ministerio de Economía y Competitividad (MINECO) for the Juan de la CiervaIncorporación program. P.R.S. thanks MINECO and the Fondo Social Europeo (FSE) for the "Promoción del talento y su Empleabilidad en I+D+i" statal program (BES-2014-069410). M.I.M. acknowledges the financial support from MINECO Grant FIS2015-69295-C3-3-P and from the María de Maeztu Programme for Units of Excellence in R\&D (Grant MDM-2014-0377). N.d.S. thanks MINECO for grants FIS2012-36113-C03, FIS2015-69295-C3-3-P, and MAT2014-58860-P and the Comunidad de Madrid (Contract No. S2013/MIT-2740). J. R. Procopio and M. T. Sevilla are acknowledged for material support. A. García-Martín is gratefully acknowledged for supporting computing facilities.

\section{REFERENCES}

[1] D. Wirtz, [Particle-Tracking Microrheology of Living Cells: Principles and Applications], (2009).

[2] T. Liu, X. Liu, D. R. Spring et al., "Quantitatively Mapping Cellular Viscosity with Detailed Organelle Information via a Designed PET Fluorescent Probe," Scientific Reports, 4, 5418 (2014).

[3] S. Suresh, J. Spatz, J. P. Mills et al., "Connections between single-cell biomechanics and human disease states: gastrointestinal cancer and malaria," Acta Biomaterialia, 1(1), 15-30 (2005).

[4] E. M. Darling, and D. Di Carlo, [High-Throughput Assessment of Cellular Mechanical Properties] Annual Reviews, Palo Alto(2015).

[5] Y.-Q. Chen, C.-Y. Kuo, M.-T. Wei et al., "Intracellular viscoelasticity of HeLa cells during cell division studied by video particle-tracking microrheology," Journal of Biomedical Optics, 19(1), 011008-011008 (2013).

[6] L. Chevry, R. Colin, B. Abou et al., "Intracellular micro-rheology probed by micron-sized wires," Biomaterials, 34(27), 6299-6305 (2013).

[7] J. S. H. Lee, P. Panorchan, C. M. Hale et al., "Ballistic intracellular nanorheology reveals ROCK-hard cytoplasmic stiffening response to fluid flow," Journal of Cell Science, 119(9), 1760-1768 (2006).

[8] S. Chen, Y. Hong, Y. Zeng et al., "Mapping Live Cell Viscosity with an Aggregation-Induced Emission Fluorogen by Means of Two-Photon Fluorescence Lifetime Imaging," Chemistry - A European Journal, 21(11), 4315-4320 (2015). 
[9] M. K. Kuimova, G. Yahioglu, J. A. Levitt et al., "Molecular Rotor Measures Viscosity of Live Cells via Fluorescence Lifetime Imaging," Journal of the American Chemical Society, 130(21), 6672-6673 (2008).

[10] M. K. Kuimova, S. W. Botchway, A. W. Parker et al., "Imaging intracellular viscosity of a single cell during photoinduced cell death," Nat Chem, 1(1), 69-73 (2009).

[11] M. S. Yousafzai, F. Ndoye, G. Coceano et al., "Substrate-dependent cell elasticity measured by optical tweezers indentation," Optics and Lasers in Engineering, 76, 27-33 (2016).

[12] S. Henon, G. Lenormand, A. Richert et al., "A new determination of the shear modulus of the human erythrocyte membrane using optical tweezers," Biophysical Journal, 76(2), 1145-1151 (1999).

[13] J. F. Berret, "Local viscoelasticity of living cells measured by rotational magnetic spectroscopy," Nature Communications, 7, 10134 (2016).

[14] Y. A. Ayala, B. Pontes, D. S. Ether et al., "Rheological properties of cells measured by optical tweezers," Bmc Biophysics, 9, (2016).

[15] A. Yao, M. Tassieri, M. Padgett et al., "Microrheology with optical tweezers," Lab on a Chip, 9(17), 2568-2575 (2009).

[16] K. C. Neuman, and S. M. Block, “Optical trapping," Review of Scientific Instruments, 75(9), 2787-2809 (2004).

[17] A. I. Bishop, T. A. Nieminen, N. R. Heckenberg et al., "Optical microrheology using rotating laser-trapped particles," Physical Review Letters, 92(19), (2004).

[18] J. Chen, and J. X. Zhao, "Upconversion Nanomaterials: Synthesis, Mechanism, and Applications in Sensing," Sensors, 12(3), 2414 (2012).

[19] G. Chen, H. Qiu, P. N. Prasad et al., "Upconversion Nanoparticles: Design, Nanochemistry, and Applications in Theranostics," Chemical Reviews, 114(10), 5161-5214 (2014).

[20] W. Fang, and Y. Wei, "Upconversion nanoparticle as a theranostic agent for tumor imaging and therapy," Journal of Innovative Optical Health Sciences, 09(04), 1630006 (2016).

[21] F. Wang, D. Banerjee, Y. Liu et al., "Upconversion nanoparticles in biological labeling, imaging, and therapy," Analyst, 135(8), 1839-1854 (2010).

[22] M. Bettinelli, L. Carlos, and X. Liu, "Lanthanide-doped upconversion nanoparticles," Physics Today, 68(9), 3844 (2015).

[23] F. Ai, Q. Ju, X. Zhang et al., "A core-shell-shell nanoplatform upconverting near-infrared light at $808 \mathrm{~nm}$ for luminescence imaging and photodynamic therapy of cancer," Scientific Reports, 5, 10785 (2015).

[24] Y. I. Park, H. M. Kim, J. H. Kim et al., "Theranostic Probe Based on Lanthanide-Doped Nanoparticles for Simultaneous In Vivo Dual-Modal Imaging and Photodynamic Therapy,” Advanced materials, 24(42), 57555761 (2012).

[25] C. Wang, L. Cheng, and Z. Liu, "Drug delivery with upconversion nanoparticles for multi-functional targeted cancer cell imaging and therapy," Biomaterials, 32(4), 1110-1120 (2011).

[26] Y. Yang, B. Velmurugan, X. Liu et al., "NIR Photoresponsive Crosslinked Upconverting Nanocarriers Toward Selective Intracellular Drug Release," Small, 9(17), 2937-2944 (2013).

[27] P. Chen, M. Song, E. Wu et al., "Polarization modulated upconversion luminescence: single particle vs. fewparticle aggregates," Nanoscale, 7(15), 6462-6466 (2015).

[28] J. Zhou, G. Chen, E. Wu et al., "Ultrasensitive Polarized Up-Conversion of Tm3+-Yb3+ Doped beta-NaYF4 Single Nanorod," Nano Letters, 13(5), 2241-2246 (2013).

[29] P. Rodriguez-Sevilla, L. Labrador-Paez, D. Wawrzynczyk et al., "Determining the 3D orientation of optically trapped upconverting nanorods by in situ single-particle polarized spectroscopy," Nanoscale, 8(1), 300-308 (2016).

[30] P. Rodríguez-Sevilla, Y. Zhang, P. Haro-González et al., "Thermal Scanning at the Cellular Level by an Optically Trapped Upconverting Fluorescent Particle," Advanced Materials, 28(12), 2421-2426 (2016).

[31] Y. Cho, S. W. Song, S. Y. Lim et al., "Spectral evidence for multi-pathway contribution to the upconversion pathway in NaYF4:Yb3+,Er3+ phosphors," Physical Chemistry Chemical Physics, 19(10), 7326-7332 (2017).

[32] [COMSOL, Multiphysics, 5.2, http://www.comsol.com].

[33] F. Borghese, P. Denti, R. Saija et al., "Radiation torque on nonspherical particles in the transition matrix formalism," Optics Express, 14(20), 9508-9521 (2006).

[34] R. M. Erb, J. Segmehl, M. Charilaou et al., "Non-linear alignment dynamics in suspensions of platelets under rotating magnetic fields," Soft Matter, 8(29), 7604-7609 (2012). 
[35] M. A. Islam, "Einstein-Smoluchowski Diffusion Equation: A Discussion," Physica Scripta, 70(2-3), 120 (2004).

[36] Y. Y. Hui, B. Zhang, Y.-C. Chang et al., "Two-photon fluorescence correlation spectroscopy of lipidencapsulated fluorescent nanodiamonds in living cells," Optics Express, 18(6), 5896-5905 (2010).

[37] N. F. Reuel, A. Dupont, O. Thouvenin et al., "Three-Dimensional Tracking of Carbon Nanotubes within Living Cells," ACS Nano, 6(6), 5420-5428 (2012). 